\title{
THE OPTICAL COUNTERPART OF M101 ULX-1
}

\author{
K. D. KUNTZ \\ Department of Physics, University of Maryland Baltimore County, \\ 1000 Hilltop Circle, Baltimore MD, 21250 \\ Robert A. Gruendi, You-Hua CHU, C.-H. Rosie Chen
Department of Astronomy, University of Mlinois at Urbana-Champaign,
1002 West Green Street, Urbana IL, 61801 \\ Martin Still ${ }^{2}$, Koji MUKaI ${ }^{2}$, ANd RichaRd F. MUShotzky \\ Laboratory for High Energy Astrophysics, \\ Code 662, NASA/GSFC, Greenbeit, MD 20771 \\ Draft version October 25, 2004
}

\begin{abstract}
We have identified the optical counterpart of the Ultra-Luminous X-ray source M101 ULX-1 (CXOKM101 J140332.74+542102), by comparing HST ACS images with Chandra ACIS-S images. The optical counterpart has $\mathrm{V}=23.75$ and colours consistent with those for a mid-B supergiant. Archival WFPC2 observations show that the source brightness is constant to within $\sim 0.1 \mathrm{mag}$. The physical association of this source with the ULX is confirmed by Gemini GMOS spectroscopic observations which show spatially unresolved He II $\lambda 4686$ and He I $\lambda 5876$ emission. These results suggest that M101 ULX-1 is a HMXB but deep spectroscopic monitoring observations are needed to determine the detailed properties of this system.

Subject headings: X-rays: individual (M101 ULX-1) - X-rays: binaries
\end{abstract}

\section{INTRODUCTION}

Ultraluminous X-ray sources (ULXs) are off-nuclear sources with $L_{\mathrm{X}}$ greater than $10^{39} \mathrm{ergs} \mathrm{s}^{-1}$, the Eddington luminosity for an accreting black hole of a few solar masses. This high $L_{\mathrm{X}}$ could either result from an overcorrection of beamed emission or be associated with an intermediate-mass black hole $\left(M_{\mathrm{BH}} \gtrsim 100 M_{\odot}\right)$. A small number of ULXs are surrounded by extended He II nebulae indicating that they are not beamed (Pakull \& Mirioni 2002), but the nature of most ULXs remains ambiguous. In fact, ULXs may not be a unified class of object, since some are found near active star formation regions (Zezas \& Fabbiano 2002) and some are in elliptical galaxies (Angelini, Loewenstein, \& Mushotzky 2001). The nature of ULXs can be ascertained if their optical counterparts can be identified and studied. To date, only a few ULXs have potential optical counterparts, and in each case multiple plausible candidates exist. No ULXs have had their optical counterparts identified definitively.

Recently, the X-ray source P98 in M101 was identified as a ULX (Pence et al. 2001; Mukai et al. 2003). This source, designated as CXOKM101 J140332.74+542102, was previously detected by the ROSAT HRI in one of four observations (source H32 of Wang, Immler, \& Pietsch 1999). Its extraordinary nature was first revealed in a 97 ks Chandra AO-1 observation when its $L_{\mathrm{X}}$ reached $1.2 \times 10^{39} \mathrm{ergs} \mathrm{s}^{-1}$ in the $0.5-2.0 \mathrm{keV}$ band (bolometric $L=3-5 \times 10^{39}$ ), qualifying its notation as M101 ULX-1. Subsequent observations show an ex-

\footnotetext{
Electronic address: kuntz@milkyway.gsfc.nasa.gov

1 Mailing address: Laboratory for High Energy Astrophysics, Code662, NASA/GSFC, Greenbelt, MD 20771

2 Universities Space Research Association
}

ponential decline with a time-scale of $\sim 200$ days and a quiescent $L_{\mathrm{X}} \sim 4 \times 10^{36} \mathrm{ergs} \mathrm{s}^{-1}$ in the $0.5-2.0 \mathrm{keV}$ band. The X-ray spectral shape of M101 ULX-1 at its extreme high state is strongly variable and can be fitted with a disk blackbody model and a power-law excess typical for black hole candidates (Mukai et al. 2003, 2005). We have continued to monitor M101 ULX-1 throughout the ongoing M101 1 Ms exposure with Chandra. During the 2004 July 5-11 observations, it entered a brief but less extreme high state with a peak $L_{\mathrm{X}} \sim 4.3 \times 10^{38} \mathrm{ergs} \mathrm{s}^{-1}$, but quickly returned to its quiescent level. The details of the X-ray observations will be reported elsewhere.

Using HST ACS data, we identified an optical counterpart. Subsequently, we obtained Gemini GMOS spectroscopic observations of this optical counterpart on 2004 July 22 , and found spatially unresolved spectral features that confirm the association of the optical counterpart with M101 ULX-1. An XMM-Newton observation less than 24 hours later confirms that the ULX had returned to a quiescent state. In this paper we describe the initial identification of the optical counterpart of M101 ULX-1 in $\S 2$, describe the spectroscopic confirmation in $\S 3$, and discuss the implications of the data in $\S 4$.

\section{OPTICAL IDENTIFICATION}

\subsection{HST ACS Observations: An ID}

The region of M101 containing ULX-1 was observed with the HST ACS on 2002 November 15 as part of a larger program to identify optical counterparts of X-ray point sources. Exposures were made in $\mathrm{F} 435 \mathrm{~W}$ (900 s), F555W (720 s), and F814W (720 s) using the CR-split mode but without dithering. The HST pointing uncertainty is on the order of $1^{\prime \prime}$. To obtain the best possible positional information, we mosaicked together 16 differ- 


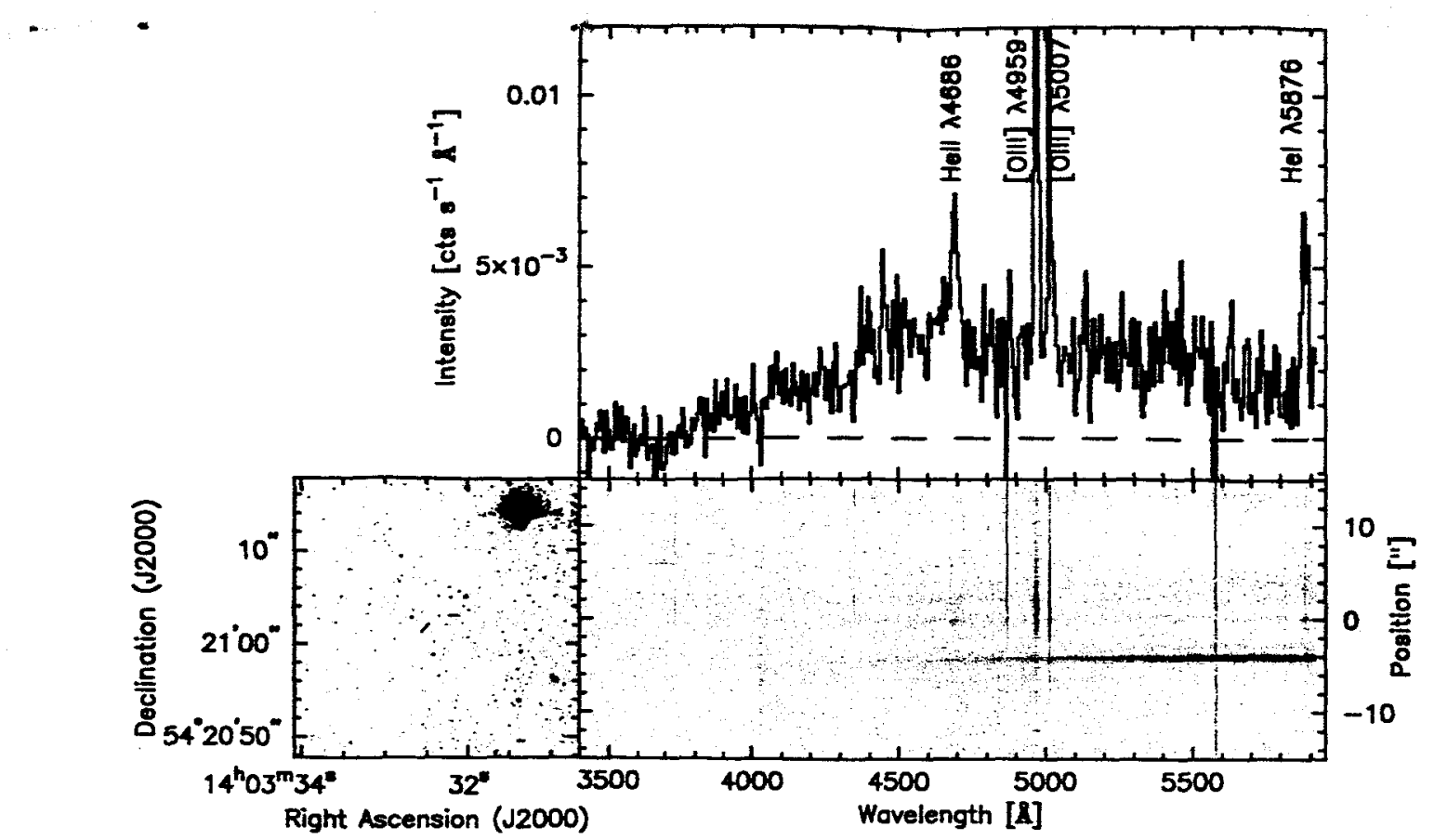

FIG. 3.- Bottom Left: The ACS F555W image of the region surrounding the ULX. Two short line segments indicate the optical counterpart of M101 ULX-1 in the center of the field. Bottom Right: Gemini GMOS spectrogram of a N-S oriented slit centered on the optical counterpart of ULX-1, at position $=0$. Note the interstellar lines of [O IM] $\lambda \lambda 3726,3729, \mathrm{H} \delta, \mathrm{H} \gamma$, and $\mathrm{H} \beta$ arise primarily north of the ULX. Top: A sky-subtracted spectrum of ULX-1 extracted with a $2^{\prime \prime}$ aperture and binned by a factor of 7 along the dispersion. The narrow interstellar and broad stellar emission lines are marked above the spectrum. The [O I] $\lambda 5577$ line is a residual sky line. The gaps between CCDs fell in the spectral regions near 4015 and $4959 \AA$, and the interpolation between the chips blurs the [O III] $\lambda 4959$ line.

sion 1.6) within IRAF, A bias from calibration observations over the previous two-week period was subtracted, cosmic rays were rejected, and a flat field was applied. The wavelength calibration and spatial distortion correction, determined from an observation of a CuAr lamp made just prior to the source observations, were applied to the observations. The five individual exposures were then combined to obtain a single deep source observation.

The position of M101 ULX-1 along the slit was verified by comparing the pattern of continuum sources with the target acquisition image and an HST ACS F555W image. The contribution due to sky background was estimated using a region of the spectrogram between $5^{\prime \prime}$ and $10^{\prime \prime}$ north of ULX-1 which contained no obvious stellar emission. Figure 3 shows the background-subtracted spectrogram from 3100 to $5900 \AA$ in the vicinity of ULX-1.

\subsection{Spectroscopic Properties}

The Gemini GMOS spectrogram in Figure 3 shows continuous spectra of stars and emission lines from ionized interstellar gas, as well as the residual [O I] sky line. The interstellar emission lines are narrow (spectrally unresolved) but spatially extended, showing intensity variations along the slit. These include the hydrogen Balmer series, He I $\lambda 3889$ (blended with $\mathrm{H} 8$ ), He I $\lambda 5876$, [O III] $\lambda \lambda 49559,5007$, and [O II] $\lambda \lambda 3726,3729$ lines. The $\left[\mathrm{O}\right.$ III] $/ \mathrm{H} \beta$ ratio is enhanced from $2^{\prime \prime} \mathrm{S}$ to $8^{\prime \prime} \mathrm{N}$ of the ULX, corresponding to $320 \mathrm{pc}$ in spatial extent and indicating a high excitation, but no He II line emission is detected in this interstellar gas. It is not clear whether ULX-1 is responsible for the ionization of this diffuse emission. The radial velocities of the interstellar $\mathrm{H} \beta$ and [O III] lines are $300 \pm 10 \mathrm{~km} \mathrm{~s}^{-1}$, consistent with the velocity of
M101.

The spectrum extracted from ULX-1 does not have a sufficient signal-to-noise ratio to show the photospheric absorption lines from the star; however, emission lines of He I $\lambda 5876$ and He II $\lambda 4686$ are clearly detected. These two spatially unresolved emission lines are easily visible in the spectrogram as well. The absence of He II interstellar emission indicates that the He II emission line must arise in the immediate vicinity of M101 ULX-1. The He I line is centered at $320 \mathrm{~km} \mathrm{~s}^{-1}$ (consistent with the velocity of M101) with a FWHM of $\sim 900 \mathrm{~km} \mathrm{~s}^{-1}$. The He II is $\sim 1200 \mathrm{~km} \mathrm{~s}^{-1}$ wide with a central velocity $V_{\text {hel }}=200-300 \mathrm{~km} \mathrm{~s}^{-1}$. The extended blue shoulder of the He II line is likely due to the C III/N III $\lambda \lambda 4634-4650$ $\AA$ Bowen flourescence blend pumped by the He II Ly $\alpha$ line. A deeper spectrum is needed to place the stellar continuum in order to identify the He II $\lambda 4686$ and other emission line features accurately.

\section{DISCUSSION}

At $\mathrm{M}_{V}=-5.55$, this system is too bright to be a LMXB (van Paradijs \& McClintock 1995). Thus we assume that the ULX is a HMXB, and that the bulk of the optical emission is due to the stellar counterpart and not the disk, as is usual for these systems. The Galactic column in this direction is $\sim 10^{20} \mathrm{~cm}^{-2}$, and the entire column through M101 is $\sim 6 \times 10^{20} \mathrm{~cm}^{-2}$, so the reddening of the optical counterpart is likely to be small. If we assume minimal reddening, the $B V I$ magnitudes and colors of the optical counterpart of ULX-1 are consistent with those of a B supergiant with a mass of 9-12 $\mathrm{M}_{\odot}$ and little reddening (see Figure 4). However, given that this star is part of a binary in which significant mass 


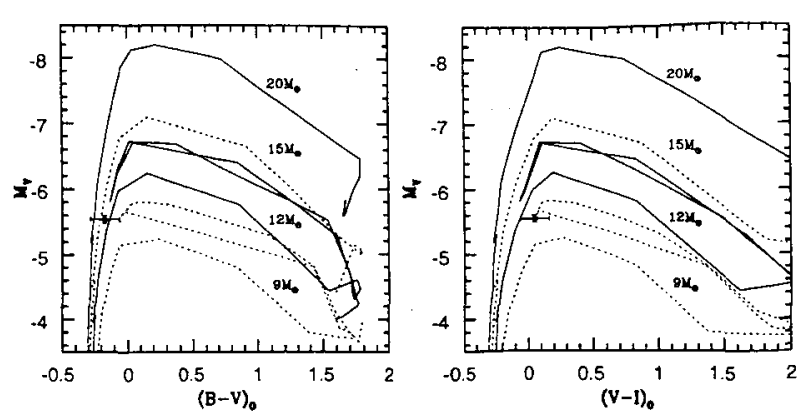

FIG. 4.- The position of the ULX in color magnitude diagrams containing the tracks of $9,12,15$, and $20 \mathrm{M}_{\odot}$. The tracks are those of Lejeune \& Schaerer (2001) and alternate between solid and dotted with mass. Comparison of our ACS photometry with the tracks of Girardi et al. (2002) produce a similar result.

transfer has occured, the true stellar mass can only be determined spectroscopically from the orbit. The quiescent X-ray flux of $10^{36} \mathrm{erg} \mathrm{s}^{-1}$ is typical of HMXB systems containing supergiants (van Paradijs \& McClintock 1995), but the X-ray emission is unusually soft.

In neither Wolf-Rayet nor normal massive stars does the combination of He II $\lambda 4686$ and He I $\lambda 5876$ emission arise with this intensity ratio or without other strong lines such as He II $\lambda 4860$ (Walborn 1980; Walborn \& Fitzpatrick 1990; Smith \& Willis 1983). Since B supergiants (assuming our classification by colour) are not hot enough to produce $\mathrm{He}$ II, the $\mathrm{He}$ II-emitting material must be photoionized by the X-ray emission from ULX-1. The absence of forbidden lines indicates a high gas density, much higher than the critical density of $\sim 10^{7} \mathrm{~cm}^{-3}$ for the [O III] $\lambda \lambda 4959,5007$ lines. The absence of $\mathrm{H}$ emission or absorption lines suggests that the emitting material is $\mathrm{H}$-deficient, or He-rich. We note however that as in the case of $4 \mathrm{U} 1700-377$, another system with He II $\lambda 4686$ and $\mathrm{He} \mathrm{I} \lambda 5876$ emission, the $\mathrm{H} \beta$ - $\epsilon$ series may be in absorption (Hutchings 1974; Blake et al. 1995). It would be difficult to detect the Balmer absorption lines in this spectrum especially in the presence of Balmer interstellar emission lines. Spectra at $\mathrm{H} \alpha$ may resolve this issue. Overall, the anomalous abundances and high density of the emitting material make an interstellar origin unlikely, and the spatial coincidence of the He II emission and the $B$ supergiant strongly suggests that they are physically associated with ULX-1.
We are not able to localize the He II and He I emission by analogy with better-understood systems. In the Her $\mathrm{X}-1$ system, the He II emission arises in the accretion disk close to the neutron star (Still et al. 1997), while in the Cyg X-1 system, the He II emission arises in either the stellar wind (Ninkov, Walker, \& Yang 1987) or at the L1 point (Aab 1983) and is accompanied by $\mathrm{H} \alpha$. The lack of large-amplitude photometric variations suggest that there were no strong ellipsoidal variations or eclipses on the timescale of the GMOS observations of M101 ULX-1.

One might use the argument of Pakull \& Angebault (1986), that the He II $\lambda 4686$ photon rate is directly proportional to the X-ray flux with $\mathrm{E}>0.054 \mathrm{keV}$, to estimate the size of the He II emission region. However, the XMM-Newton spectrum taken on the following day has only 27 counts in the MOS cameras and 78 counts in the PN camera (after background subtraction), and the counts are all at $0.3<E<0.7 \mathrm{keV}$. Assuming the same amount of intrinsic absorption as was seen in the AO-1 high state, several different models can be fitted to the XMM-Newton data. All suggest that the $\lambda 4686$ photon rate of $2.4 \times 10^{-5}$ photons $\mathrm{cm}^{-2} \mathrm{~s}^{-1}$ corresponds to $1 \%-10 \%$ of the He II ionizing flux. The covering fraction is very sensitive to the value assumed for the intrinsic absorption and other higher but reasonable values of absorption lead to lower covering fractions.

He II $\lambda 4686$ emission is the classical "hallmark" of X-ray binary optical counterparts (Pakull \& Angebault 1986), the "smoking gun" required to confirm counterparts, even though it is sometimes not clear where in the system the emission occurs. A deep, high-quality spectrum of the B supergiant is needed to accurately determine the spectral type and mass of this counterpart. The He emission lines need to be monitored for orbital velocity variation in order to localize their emission, confirm the HMXB nature of ULX-1, and determine the mass of the compact companion.

We thank M. Mountain and J.-R. Roy for granting Gemini DD time and P. Gomez for executing the GMOS observations, I. Jorgensen, M. Bergman, and R. C. Smith for advising us on the calibration and reduction of GMOS data and N. Walborn for useful discussions regarding spectral classification. This program is supported by the Chandra grant SAO GO-5600587.

\section{REFERENCES}

Aab, O. E. 1983, Sov. Astron. Lett., 9, 606

Angelini, L., Loewenstein, M., \& Mushotzky, R. F. 2001, ApJ, 557, L35

Blake, C. C., Marlborough, J. M., Walker, G. A. H., \& Fahlman, G. G. 1995, AJ, 109, 2698

Girardi, L., Bertelli, G., Bressan, A., Chiosi, C., Groenewegen, M. A. T., Marigo, P., Salasnich, B., \& Weiss, A. 2002, A\&A, 391, 195

Holtzman, J. A., Burrows, C. J., Casertano, S., Hester, J. J., Trauger, J. T., \& Worthey, A. M. W. G. 1995a, PASP, 107, 1065

Holtzman, J. A., et al. 1995b, PASP, 107, 156

Hutchings, J. B. 1974, ApJ, 192, 677

Lejeune, T., \& Schaerer, D. 2001, A\&A, 366, 538

Mukai, K., et al. 2005, in preparation

Mukai, K., Pence, W. D., Snowden, S. L., \& Kuntz, K. D. 2003, ApJ, 582, 184

Ninkov, Z., Walker, G. A. H., \& Yang, S. 1987, ApJ, 321, 438
Pakull, M. W., \& Angebault, L. P. 1986, Nature, 322, 511

Pakull, M. W., \& Mirioni, L. 2002, in Winds, Bubbles, and Explosions, ed. S. J. Arthur \& W. J. Henney, 197

Pence, W. D., Snowden, S. L., Mukai, K., \& Kuntz, K. D. 2001, ApJ, 561, 189

Smith, L. J., \& Willis, A. J. 1983, A\&AS, 54, 229

Stetson, P. B., et al. 1998, ApJ, 508, 491

Still, M. D., Quaintrell, H., Roche, P. D., \& Reynolds, A. P. 1997, MNRAS, 292, 52

van Paradijs, J., \& McClintock, J. E. 1995, in X-ray Binaries, ed. W. H. G. Lewin, J. van Paradijs, \& E. P. J. van den Heuvel, 58 Walborn, N. R. 1980, ApJS, 44, 535

Walborn, N. R., \& Fitzpatrick, E. L. 1990, PASP, 120, 379

Wang, Q. D., Immler, S., \& Pietsch, W. 1999, ApJ, 523, 121

Zezas, A., \& Fabbiano, G. 2002, ApJ, 577, 726 